VOL. $12(1975), 277-282$.

\title{
On a class of finite
}

\section{soluble groups}

\section{G. Karpilovsky}

Let the group $T$ be the direct product of groups $S_{i}$ $(i=1, \ldots, r)$ where for a given group $A_{i}, S_{i}$ is the direct product of $n_{i}$ factors $A_{i} \times A_{i} \times \ldots \times A_{i}$. Let $B$ be a group that has a faithful permutation representation $\Gamma_{i}$ of degree $n_{i}$ $(i=1, \ldots, r)$. Consider $G$, the split extension of $T$ by $B$ defined by letting $B$ act on $T$ as follows.

Each $S_{i}$ is normal in $G$. If $\left(a_{1}, \ldots, a_{n_{i}}\right) \in S_{i}$ and $b \in B$ then $\left(a_{1}, \ldots, a_{n_{i}}\right)^{b}=\left(a_{\alpha_{1}}, a_{\alpha_{2}}, \ldots, a_{\alpha_{n_{i}}}\right)$ where $\Gamma_{i}(b)=\left(\begin{array}{cccc}\alpha_{1} & \alpha_{2} & \cdots & \alpha_{n_{i}} \\ 1 & 2 \ldots & n_{i}\end{array}\right)$. It is proved that if $T$ is an $M$-group and all subgroups of $B$ are $M$-groups, then $G$ is an $M$-group. This is a generalisation of a result of Gary M. Seitz, Math. Z. 110 (1969), 101-122, who proved the particular case where $r=1$ and $\Gamma_{1}$ is the regular representation of $B$.

A finite group $G$ is an $M$-group if each irreducible complex character of $G$ is induced from a linear character of a subgroup of $G$. Some of the difficulties involved in studying $M$-groups are indicated by a result of Dade which states that any finite soluble group can be embedded

Received 6 December 1974. 
in an $M$-group [3]. Seitz sharpened Dade's result by proving that a finite soluble group can be embedded in an $M$-group with the same derived length. This statement is a corollary of the following result of seitz [4]:

Let $A$ be an $M$-group and suppose $B$ is a group all of whose subgroups are $M$-groups. Then $A$ wr $B$, the wreath product of $A$ with $B$ is an $M$-group.

The aim of this paper is to generalise this result of Seitz. We shall use the following notation:

aut $G$ is the group of automorphisms of the group $G$;

ker $\chi$ is the kernel of the character $x$;

an $\tilde{M}$-group is a group all of whose subgroups are $M$-groups;

if $H$ is a subgroup of the group $G$ and $X$ a character of $H$ then $\chi^{G}$ is the character of $G$ induced from $\chi$; $N_{G}(\chi)$ is the stabilizer of $X$ in $G$;

$\psi \downarrow H$ is the restriction of a character $\psi$ of a group $G$ to its subgroup $H$;

$a^{b}=b^{-1} a b$.

LEMMA 1 [2]. Let $H \triangleleft G, X$ an irreducible character of $H$ which has an extension $\hat{x}$ to $T=N_{G}(\chi)$. Then $\chi^{G}=\sum_{\omega} \omega(I)(\omega \hat{x})^{G}$ where the sum muns over the irreducible characters of $T / H$. Each character $(\hat{\omega \chi})^{G}$ is irreducible and $\left(\omega_{1} \hat{x}\right)^{G}=\left(\omega_{2} \hat{x}\right)^{G}$ implies $\omega_{1}=\omega_{2}$.

LEMMA 2 [1]. Suppose $H \triangleleft G$. If $H$ is abelian and complemented in $G$, then each irreducible character of $H$ extends to its stabilizer.

LEMMA 3. Let $G=A \cdot B \quad(A \triangleleft G, A \cap B=1)$. Then each linear character of $A$ extends to its stabilizer.

Proof. Let $T=N_{G}(X)$ be the stabilizer of the linear character $\chi$ of $A$. Then $T=A \cdot B_{0} \quad\left(B_{0}=B \cap T\right)$. If $\operatorname{ker} x=K \quad(K \triangleleft T)$ then $T / K=(A / K) \cdot\left(B_{0} K / K\right)$ (semidirect product) and $A / K$ is cyclic. Now use 
Lemma 2.

It is clear that not every extension of an $M$-group by an $\tilde{M}$-group is an $h$-group. We formulate one sufficient condition for such an extension to be an M-group.

THEOREM 1. Let $G=A \cdot B \quad(A \triangleleft G, A \cap B=1)$ where $A$ is an $M$-group and $B$ is an $\tilde{M}$-group. If for each irreducible character $x=\phi^{A}$ of the group $A$ where $\phi$ is a linear character of a group $H \subseteq A$, $N_{G}(\chi) \cap B \subseteq N_{G}(\phi)$, then $G$ is an M-group.

Proof. Let $N_{G}(X)=T$ and $B_{0}=T \cap B$; then $T=A \cdot B_{0}$. In view of $B_{0} \subseteq N_{G}(\phi)$ the stabilizer of the character $\phi$ in the group $S=H B_{0}$ $\left(H \triangleleft S, H \cap B_{0}=1\right)$ is $S$. Thus the linear character $\phi$ of the group $H$ can be extended to a linear character $\hat{\phi}$ of the group $S$ (see Lemma 3 ). Now $\hat{\phi}^{T} \downarrow A=\left(\hat{\phi} \downarrow H B_{0} \cap A\right)^{A}=(\hat{\phi} \downarrow H)^{A}=\phi^{A}=\chi$. (We have used the subgroup theorem [1] and the fact that $\left.\left(H B_{0}\right) A=T.\right)$ Thus the character $X$ has an extension to the irreducible monomial character $\hat{\phi}^{T}$ of the group $T=N_{G}(X)$. Now using Lemma I we have $\chi^{G}=\sum_{\omega} \omega(1)\left(\omega \hat{\phi}^{T}\right)^{G}$. Since $B$ is an $\tilde{M}_{\text {-group, the group }} T / A \cong B_{0} \subseteq B$ is an $M$-group, and therefore if $\omega$ is any irreducible character of $T / A$ then $\omega=\psi^{T}$ where $\psi$ is a linear character of the group $R \quad(A \subseteq R \subseteq T)$. Further

$$
\left(\omega \hat{\phi}^{T}\right)^{G}=\left(\psi^{T} \cdot \hat{\phi}^{T}\right)^{G}=\left[\left(\psi\left(\hat{\phi}^{T} \downarrow R\right)\right)^{T}\right]^{G}=\left[\psi\left(\hat{\phi}^{T} \downarrow R\right)\right]^{G}
$$

But $\hat{\phi}^{T} \downarrow R=\left(\hat{\phi} \downarrow H B_{0} \cap R\right)^{R}$ and hence

$$
\left(\omega \hat{\phi}^{T}\right)^{G}=\left[\psi\left(\hat{\phi} \downarrow H B_{0} \cap R\right)^{R}\right]^{G}=\left[\left[(\psi \hat{\phi})+H B_{0} \cap R\right]^{R}\right]^{G}=\left[(\psi \hat{\phi}) \downarrow H B_{0} \cap R\right]^{G},
$$

which proves the theorem.

Now let $T=A \times A \times \ldots \times A \quad(n$ factors $)$ and assume that a group $B$ admits a faithful representation $\Gamma$ by permutations of degree $n$. Let $a=\left(a_{1}, a_{2}, \ldots, a_{n}\right) \quad\left(a_{i} \in A\right)$ be any element of $T$ and $b$ any element 
of $B$ with $\Gamma(b)=\left(\begin{array}{lll}\alpha_{1} \alpha_{2} & \ldots & \alpha_{n} \\ 1 & 2 \ldots & n\end{array}\right)$. Then

$$
\phi_{b}(a)=\left(a_{\alpha_{1}}, a_{\alpha_{2}}, \ldots, a_{\alpha_{n}}\right)
$$

is an automorphism of the group $T$ and the mapping $\psi: B \rightarrow$ aut $T$ where, for each $b \in B, \psi(b)=\phi_{b} \in$ aut $T$, is a homomorphism of the group $B$ into the group aut $T$. We shall call the automorphism $\phi_{b}$, defined by (1), the automorphism corresponding to $\Gamma$.

$$
\text { Consider the group } G=T \cdot B \quad(T \triangleleft G, T \cap B=1) \text { where } a^{b}=\phi_{b}(a)
$$

$(a \in T, b \in B)$. Then this group is isomorphic to the wreath product of the group $T$ with the permutation group $\Gamma(B)$. When $\Gamma$ is the regular representation of the group $B$ we have the standard wreath product

$T$ wr $B$. The following theorem considers a more general type of group.

THEOREM 2. Let $T=S_{1} \times S_{2} \times \ldots \times S_{r}$ where $S_{i}=A_{i} \times A_{i} \times \ldots \times A_{i}\left(n_{i}\right.$ factors; $\left.i=1,2, \ldots, r\right)$ and let $B$ be a group which admits a farthful representation $\Gamma_{i}$ by permutations of degree $n_{i}(i=1,2, \ldots, r)$. Let $G$ be the split extension of $T$ by $B$, where each $S_{i}$ is invariant under $B$, and the action of $b \in B$ on $S_{i}$ is given by the automorphism determined by $\Gamma_{i}(b)$ according to the mule given in (1). Then if $T$ is an $M$-group and $B$ is an $\tilde{M}$-group then $G$ is an M-group.

Proof. First of all consider the case when $r=1$. Then $G=T B$ $(T \triangleleft G, T \cap B=1), T=A \times A \times \ldots \times A \quad(n$ factors $)$. If $b \in B$ is any

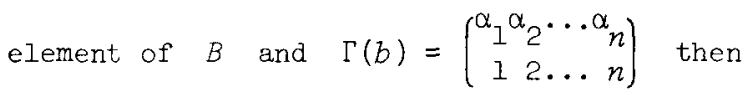

$$
a^{b}=\left(a_{\alpha_{1}}, a_{\alpha_{2}}, \ldots, a_{\alpha_{n}}\right)
$$

where $a=\left(a_{1}, a_{2}, \ldots, a_{n}\right)$ is any element of $T\left(a_{i} \in A\right)$. Let $x=x_{1} x_{2} \cdots x_{n}$ be any irreducible character of $T$. Thus $x(a)=x_{1}\left(a_{1}\right) x_{2}\left(a_{2}\right) \cdots x_{n}\left(a_{n}\right)$ and $x_{i}$ is an irreducible character of $A$ 
$(i=1,2, \ldots, n)$. Now since $A$ is an $M$-group, $x_{i}=\psi_{i}^{A}$, where $\psi_{i}$ is a linear character of some $H_{i} \subseteq A \quad(i=1,2, \ldots, n)$. Now $\psi=\psi_{1} \psi_{2} \cdots \psi_{n}$ is a linear character of the group $H=H_{1} \times H_{2} \times \ldots \times H_{n} \subseteq T$ and $x=\psi^{T}$. In view of (2), $x\left(a^{b}\right)=x_{1}\left(a_{\alpha_{1}}\right) x_{2}\left(a_{\alpha_{2}}\right) \ldots x_{n}\left(a_{\alpha_{n}}\right)$, and $x\left(a^{b}\right)=x(a)$ implies $x_{i}=x_{\alpha_{i}}$ $(i=1,2, \ldots, n)$. Thus we can assume that $H_{i}=H_{\alpha_{i}}$ and $\psi_{i}=\psi_{\alpha_{i}}$ $(i=1,2, \ldots, n)$. Now $N_{G}(x)=T B_{0}\left(B_{0}=N_{G}(x) \cap T\right)$, and for each $h=\left(h_{1}, h_{2}, \ldots, h_{n}\right) \in H \quad\left(h_{i} \in H_{i} ; i=1,2, \ldots, n\right), b \in B_{0}$, we have $h^{b}=\left(h_{\alpha_{1}}, \ldots, h_{\alpha_{n}}\right)$, where $b \rightarrow\left(\begin{array}{c}\alpha_{1} \alpha_{2} \ldots \alpha_{n} \\ 12 \ldots .\end{array}\right)$ and so $b^{-1} H b \subseteq H$. Moreover, if $\psi(h)=\psi_{1}\left(h_{1}\right) \psi_{2}\left(h_{2}\right) \ldots \psi_{n}\left(h_{n}\right)$ then $\psi\left(h^{b}\right)=\psi_{1}\left(h_{\alpha_{1}}\right) \ldots \psi_{n}\left(h_{\alpha_{n}}\right)=\psi_{\alpha_{1}}\left(h_{\alpha_{1}}\right) \ldots \psi_{\alpha_{n}}\left(h_{\alpha_{n}}\right)=\psi$. Hence

$$
N_{G}(\chi) \cap B \subseteq N(\psi) \cap B
$$

This proves Theorem 2 for the case $r=1$ (by applying Theorem I). Now consider the general case. Let $x=x_{1} x_{2} \cdots x_{p}$ be any irreducible character of $T$, where $x_{i}$ is an irreducible character of $S_{i}$ $(i=1,2, \ldots, r)$. Further, for some linear character $\psi_{i}$ of the group $H_{i} \subseteq S_{i}, \quad \chi_{i}=\psi_{i}^{S_{i}} \quad(i=1,2, \ldots, r)$. As above $\psi=\psi_{1} \psi_{2} \cdots \psi_{r}$ is a linear character of $H=H_{1} \times H_{2} \times \ldots \times H_{r}$ and $\chi=\psi^{T}$. For each group $G_{i}=S_{i} B \quad\left(S_{i} \triangleleft G_{i} ; S_{i} \cap B=1, i=1, \ldots, r\right)$, we use the formula (3) to obtain $N_{G_{i}}\left(x_{i}\right) \cap B \subseteq N_{G_{i}}\left(\psi_{i}\right) \cap B$. Hence

$$
\bigcap_{i=1}^{r}\left[N_{G_{i}}\left(\chi_{i}\right) \cap B\right] \subseteq \bigcap_{i=1}^{r}\left[N_{G_{i}}\left(\psi_{i}\right) \cap B\right]
$$

Since $B_{i} \triangleleft G \quad(i=1,2, \ldots, r)$, 


$$
N_{G}(\chi) \cap B=\bigcap_{i=1}^{s}\left[N_{G}\left(\chi_{i}\right) \cap B\right]=\bigcap_{i=1}^{s}\left[N_{G}\left(\chi_{i}\right) \cap B\right] .
$$

Now using the formula (4) we have

$$
N_{G}(\chi) \cap B \subseteq \bigcap_{i=1}^{r}\left[N_{G}\left(\psi_{i}\right) \cap B\right] \subseteq N_{G}(\psi) \cap B
$$

In view of Theorem I this completes the proof.

REMARK. The result of Seitz [4] is a particular case of Theorem 2 for $r=1$, and $\Gamma_{1}$ the regular representation of the group $B$.

\section{References}

[1] Charles W. Curtis, Irving Reiner, Representation theory of finite groups and associative algebras (Pure and Applied Mathematics, 11. Interscience [John Wiley \& Sons], New York, London, 1962).

[2] P.X. Gallagher, "Group characters and normal Hall subgroups", Nagoya Math. J. 21 (1962), 223-230.

[3] B. Huppert, Endliche Gruppen I (Die Grundlehren der mathematischen Wissenschaften, 134. Springer-Verlag, Berlin, Heidelberg, New York, 1967).

[4] Gary M. Seitz, "M-groups and the supersolvable residual", Math. 2. 110 (1969), 101-122.

[5] David L. Winter and Paul F. Murphy, "Groups all of whose subgroups are M-groups", Math. Z. 124 (1972), 73-78.

School of Mathematics,

University of New South Wales,

Kensington,

New South Wales. 